\title{
Unexplained iron deficiency in idiopathic and heritable pulmonary arterial hypertension
}

\author{
Elaine Soon, ${ }^{1,2}$ Carmen M Treacy, ${ }^{1}$ Mark R Toshner, ${ }^{1}$ Robert MacKenzie-Ross, ${ }^{1}$ \\ Vijay Manglam, ${ }^{3}$ Mark Busbridge, ${ }^{4}$ Mark Sinclair-McGarvie, ${ }^{5}$ Jayantha Arnold, ${ }^{3}$ \\ Karen K Sheares, ${ }^{1}$ Nicholas W Morrell, ${ }^{2}$ Joanna Pepke-Zaba ${ }^{1}$
}

\begin{abstract}
- Additional tables are published online only. To view these files please visit the journal online (http://thorax.bmj. com).

${ }^{1}$ Pulmonary Vascular Diseases Unit, Papworth Hospital NHS

Trust, Papworth Everard, Cambridgeshire, UK

${ }^{2}$ Department of Medicine, University of Cambridge, Addenbrookes' Hospital, Cambridge, UK

${ }^{3}$ Department of

Gastroenterology, University of Buckingham, Ealing Hospital

NHS Trust, Southall, UK

${ }^{4}$ Department of Clinical

Biochemistry, Charing Cross

Hospital, Imperial College

Healthcare NHS Trust, London, UK

${ }^{5}$ Institute of Actuaries, Staple Inn Hall, High Holborn, London UK
\end{abstract}

\section{Correspondence to}

Dr Joanna Pepke-Zaba,

Pulmonary Vascular Diseases

Unit, Papworth Hospital NHS

Trust, Papworth Everard,

Cambridgeshire CB3 8RE, UK:

joanna.pepkezaba@papworth. nhs.uk

Received 26 July 2010 Accepted 6 January 2011

Published Online First

5 February 2011

\begin{abstract}
Background Anaemia is common in left heart failure and is associated with a poorer outcome. Many patients with pulmonary arterial hypertension (PAH) are anaemic or iron-deficient. This study was performed to investigate the prevalence of iron deficiency in $\mathrm{PAH}$ and to identify possible causes.

Methods All patients with idiopathic or heritable $\mathrm{PAH}$ diagnosed in 1995-2008 were identified. Controls were selected from patients with chronic thromboembolic pulmonary hypertension (CTEPH). Full blood counts were examined and any abnormality was investigated. Patients were excluded if they had a cause for iron deficiency. The prevalence study was based on 85 patients with idiopathic PAH and 120 with CTEPH. A separate group of 20 patients with idiopathic PAH and 24 with CTEPH with matching haemodynamics were prospectively investigated for serum factors affecting iron metabolism.
\end{abstract}

Results The prevalence study identified a point prevalence of unexplained iron deficiency of $50 \%$ in premenopausal women with idiopathic PAH compared with $8 \%$ in premenopausal women with CTEPH $(p=0.002) ; 14 \%$ in postmenopausal women with idiopathic PAH compared with $6 \%$ in postmenopausal women with CTEPH $(p=0.16) ; 28 \%$ in men with idiopathic PAH men compared with $2 \%$ in men with CTEPH $(p=0.002)$; and $60 \%$ in patients with heritable PAH. The serum study showed that patients with idiopathic $\mathrm{PAH}$ had lower serum iron and transferrin saturations than those with CTEPH. Interleukin-6 levels correlated with iron levels $(r=-0.6, p=0.006)$ and transferrin saturations $(r=-0.68, p=0.001)$ in idiopathic PAH but not in CTEPH. Conclusions The prevalence of unexplained iron deficiency is significantly higher in idiopathic $\mathrm{PAH}$ than in CTEPH. This may be linked to interleukin-6.

\section{INTRODUCTION}

Pulmonary arterial hypertension (PAH) is a group of rare conditions characterised by increased pulmonary artery (PA) pressures and pulmonary vascular resistance which lead to progressive right ventricular failure and death. A range of aetiologies cause $\mathrm{PAH}$, of which congenital heart disease has a well-known association with iron deficiency. ${ }^{12}$ This is thought to be the result of right-to-left shunts creating a state of chronic hypoxia. Anaemia and iron deficiency are common in left heart failure and have been shown to be associated with worse symptoms and increased mortality. ${ }^{3-6}$ Iron replacement in these patients improves symptoms and functional capacity. ${ }^{7}$
We have observed high levels of unexplained iron deficiency in our patients with idiopathic $\mathrm{PAH}$, particularly in those with heritable PAH, mainly caused by mutations in the bone morphogenetic protein type 2 receptor (BMPR2). ${ }^{8} 9$ Hepcidin which is the principal hormone governing iron homeostasis, has recently been shown to be critically influenced via a bone morphogenetic protein-dependent pathway. ${ }^{10} 11$ This prompted us to examine formally the prevalence of iron deficiency in idiopathic and heritable $\mathrm{PAH}$ and to investigate possible underlying causes. The demonstration of significant iron deficiency in patients with $\mathrm{PAH}$ may have important consequences since iron replacement may be expected to improve exercise tolerance $e^{12}$ and reduce oxidative stress. ${ }^{13}$ In addition, iron replacement has been shown to reduce PA pressure in subjects with hypoxic PAH. ${ }^{14}$

\section{METHODS \\ Subjects}

All patients diagnosed with idiopathic and heritable PAH from 1995 to $2008(n=120)$ were identified from the database at Papworth Hospital NHS Trust, Cambridge, UK. The definition of idiopathic $\mathrm{PAH}$ includes the following: mean PA pressure $>25 \mathrm{~mm} \mathrm{Hg}$ with a pulmonary capillary wedge pressure of $<15 \mathrm{~mm} \mathrm{Hg}$ and no other identifiable cause for PAH being present (Dana Point classification class 1a). ${ }^{15}$ Twenty-two patients were excluded on the basis of a pre-existing reason for iron deficiency including gastric ulceration or major surgery (eg, transplant) within a year of developing iron deficiency. Patients with idiopathic PAH with secondary shunts (ie, demonstrable flow via a patent foramen ovale) were included.

All patients with distal chronic thromboembolic pulmonary hypertension (CTEPH) and proximal CTEPH who had not undergone pulmonary endarterectomy $(n=150)$, who were diagnosed from 2000 to 2006 and originated from the Papworth catchment area (and therefore accessible for follow-up), were extracted from the database and screened for eligibility as controls. CTEPH was defined as pulmonary hypertension due to the presence of chronic thromboembolism within the pulmonary vasculature (Dana Point classification class 4). ${ }^{15}$ Twenty of the 150 patients with CTEPH were excluded because of comorbidities likely to cause iron deficiency or conditions making an accurate assessment of iron status difficult. In all cases the underlying diagnosis was confirmed at 
a multidisciplinary meeting of experienced physicians, radiologists and surgeons.

Haemodynamics were measured by right heart catheterisation. Values shown were taken from the study nearest the time of diagnosis of iron deficiency (for deficient patients) or the latest right heart catheter. The median time between right heart catheterisation and screening for iron deficiency was 0.8 years (IOR $0-2$ ). We also examined the following factors:

1. the proportion of patients who were formally anticoagulated;

2. the proportion of patients on endothelin receptor antagonists as these drugs have been associated with anaemia ${ }^{16} 17$ (although this is not thought to be due to blood loss); and

3. the proportion of patients with a baseline oxygen saturation of $<95 \%$ on air or needing oxygen to maintain saturations of $>95 \%$.

\section{Study design}

The two groups of patients being compared (PAH and CTEPH) were identified retrospectively from the patient database in the Pulmonary Vascular Diseases Unit at Papworth Hospital. Patients with CTEPH were chosen as controls because (1) they suffer from a chronic illness of comparable severity and (2) to lessen the potential confounding effects of anticoagulation, hypoxia and endothelin receptor antagonist use.
All patients were screened for exclusion criteria, which included the presence of comorbidities likely to cause iron deficiency (eg, gastric ulceration within a year of diagnosis of iron deficiency) or the presence of conditions rendering accurate assessment of iron status difficult (eg, haematological conditions requiring transfusion, full details in table 1 in online supplement). As part of their routine care, all patients had serial full blood counts. Every full blood count was checked and patients with abnormalities in haemoglobin levels, mean corpuscular volume and/or mean cell haemoglobin underwent formal iron studies either at Papworth or at their referring hospital. These included iron, ferritin, transferrin levels and saturation and C reactive protein (CRP).

An iron-deficient state was defined as decreased ferritin levels $(<10 \mu \mathrm{g} / \mathrm{l})$ with an elevated or normal transferrin ${ }^{18}$ (normal range $2.2-4.0 \mathrm{~g} / \mathrm{l}$ ) and a normal CRP level (further details in online supplement). A number of patients had microcytic anaemias but also had high concurrent CRP levels. One of the key iron indices measured was ferritin, the main storage and transport protein for iron. It is also an acute phase reactant and is elevated in inflammatory and infective conditions. Hence, in these situations ferritin does not accurately reflect iron status. The protocol for these patients was to screen for infection, treat if there was clinical suspicion and then to repeat iron studies on a separate occasion. Some patients had a persistent low-level

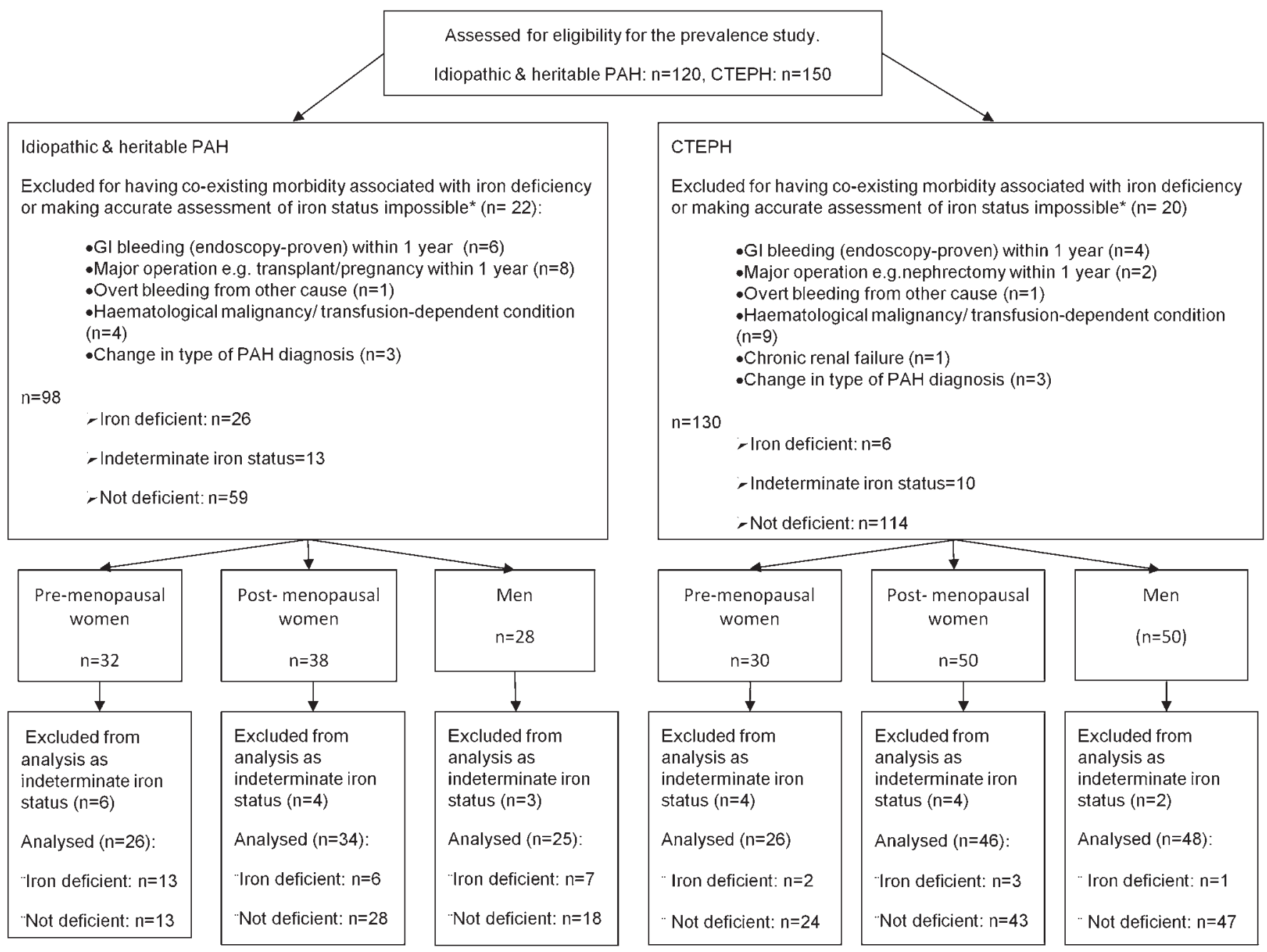

Figure 1 Flow chart showing the selection of patients with PAH and CTEPH for inclusion in the prevalence study. ${ }^{*}$ Additional details available in table 1 in the online supplement. PAH, pulmonary arterial hypertension; CTEPH, chronic thromboembolic pulmonary hypertension. 
Table 1 Baseline characteristics and haemodynamics of all patients in prevalence study

\begin{tabular}{|c|c|c|c|c|c|c|c|c|}
\hline Aetiology/gender & $n$ & Age (years) & RAP (mm Hg) & mPAP (mm Hg) & CI $\left(\mathrm{l} / \mathrm{m}^{2}\right)$ & PVR (dyne.cm-5) & 6MWD (m) & NYHA II/III/IV \\
\hline IPAH all & 85 & $51.2(16.7)^{*}$ & $9.5(5.4)$ & $52.0(13.4)^{*}$ & $2.0(0.6)$ & $981(462)^{*}$ & $320(117)^{*}$ & $11 / 64 / 10$ \\
\hline CTEPH all & 120 & $60.3(16.8)$ & $11.1(6.3)$ & $46.8(10.5)$ & $2.1(0.6)$ & $801(393)$ & $253(117)$ & $9 / 83 / 28$ \\
\hline IPAH premenopausal women & 26 & $37.7(7.9)$ & $9.2(5.9)$ & $53.3(12.0)^{*}$ & $2.0(0.6)$ & $986(321)^{*}$ & 342 (99) & $4 / 21 / 1^{*}$ \\
\hline IPAH postmenopausal women & 34 & $64.5(9.5)^{*}$ & $9.8(5.9)$ & $49.2(12.5)$ & $2.0(0.5)$ & $952(464)$ & $278(107)^{*}$ & $1 / 26 / 7$ \\
\hline CTEPH postmenopausal women & 46 & $69.4(9.3)$ & $9.5(5.4)$ & $48.0(10.2)$ & $2.1(0.6)$ & $902(427)$ & $202(107)$ & $0 / 29 / 17$ \\
\hline IPAH men & 25 & $47.3(18.1)^{*}$ & $9.3(3.9)$ & $54.5(15.6)^{*}$ & $2.0(0.6)$ & $1014(583)^{*}$ & $343(136)$ & $6 / 17 / 2$ \\
\hline
\end{tabular}

${ }^{*} \mathrm{p}<0.05$ vs corresponding CTEPH group.

BMPR2, bone morphogenetic protein receptor type 2; CTEPH, chronic thromboembolic pulmonary hypertension; IPAH, idiopathic pulmonary arterial hypertension; mPAP, mean pulmonary artery pressure; PVR, pulmonary vascular resistance.

increase in CRP. After two or more attempts at iron screening, these patients were classified as being of indeterminate status and excluded from further analyses.

Patients found to be iron-deficient were referred back to their local hospitals for further investigation. The type of investigation initiated was left to the clinical judgement of the local medical team but included gynaecological, gastroenterological and haematological review as appropriate. All patients with an identifiable cause of iron deficiency were removed. A modified CONSORT diagram is shown in figure 1 to clarify the selection process. The final analysis included a total of 85 patients with PAH and 120 with CTEPH.

A separate group of 20 patients with idiopathic PAH and 24 with CTEPH were sampled prospectively from June to December 2009 for iron indices, hepcidin and interleukin (IL)-6 (the latter being known regulators of iron metabolism). ${ }^{19-22}$ Hepcidin was measured using a previously validated immunoassay. $^{23}$ The iron status of these patients was previously unknown. For every patient with PAH sampled, a patient with CTEPH whose haemodynamic indices were the closest match was chosen as a control from among the inpatients admitted the same week. Four extra patients with CTEPH were included to ensure an equivalent gender distribution.

\section{Analysis and statistics}

For the prevalence study, patients were analysed as a whole and also divided into the following groups: (1) premenopausal women; (2) postmenopausal women (defined as 12 consecutive months without having a period); and (3) men.
This was necessary because the prevalence of iron deficiency varies widely between these groups. ${ }^{24} 25$ In the general population, iron deficiency is much more common in premenopausal women due to the demands of menstruation, pregnancy and lactation. Patients with heritable PAH were also analysed as a subset.

Descriptive statistical analyses were carried out on the study population of 85 patients with PAH and $120 \mathrm{CTEPH}$ controls using GraphPad Version 5. Initially the characteristics of all patients with idiopathic PAH and CTEPH were compared as well as the three subgroups. The point prevalence of iron deficiency was then calculated for all patients with idiopathic PAH and CTEPH as well as for the three subgroups. Finally, the characteristics of the iron-deficient and non-deficient patients with idiopathic PAH were compared. Continuous variables are expressed as mean \pm SD and compared using the Mann-Whitney test (for non-parametric data) and the unpaired t test (for parametric data). Categorical data were compared using the Fisher exact test. Statistical significance was inferred at $p<0.05$.

Multivariate models were used to analyse the probability of iron deficiency (the response variable) with respect to the following explanatory variables: cause of pulmonary hypertension (ie, IPAH or CTEPH), sex and menopausal status, age, right atrial pressure, mean PA pressure, cardiac index, NYHA class and 6-minute walking distance (6MWD). For all models considered, the model structure was a generalised linear model with logistic link function (see further details in online supplement).

For the serum study the characteristics of the 20 patients with IPAH and 24 with CTEPH were compared. The iron indices, IL-6 and hepcidin levels were then compared using the

Table 2 Comparison of characteristics which may predispose to iron deficiency

\begin{tabular}{llll}
\hline Aetiology/gender & $\begin{array}{l}\text { Proportion on } \\
\text { anticoagulation }\end{array}$ & Proportion on ERAs & $\begin{array}{l}\text { Proportion with } \\
\text { saturation }<95 \% \text { on air }\end{array}$ \\
\hline IPAH all & $67 / 85(78.8 \%)$ & $30 / 85(35.3 \%)$ & $35 / 85(41.2 \%)$ \\
CTEPH all & $120 / 120(100.0 \%)$ & $57 / 120(47.5 \%)$ & $60 / 120(50.0 \%)$ \\
& $\mathrm{p}<0.001$ & $\mathrm{p}=0.09$ & $\mathrm{p}=0.26$ \\
IPAH pre-menopausal women & $22 / 26(84.6 \%)$ & $13 / 26(50.0 \%)$ & $9 / 26(34.6 \%)$ \\
CTEPH pre-menopausal women & $26 / 26(100.0 \%)$ & $9 / 26(34.6 \%)$ & $7 / 26(26.9 \%)$ \\
& $\mathrm{p}=0.11$ & $\mathrm{p}=0.4$ & $\mathrm{p}=0.76$ \\
IPAH post-menopausal women & $27 / 34(79.4 \%)$ & $8 / 34(23.5 \%)$ & $14 / 34(41.2 \%)$ \\
CTEPH post-menopausal women & $46 / 46(100.0 \%)$ & $29 / 46(63.0 \%)$ & $30 / 46(65.2 \%)$ \\
& $\mathrm{p}=0.002$ & $\mathrm{p}<0.001$ & $\mathrm{p}=0.04$ \\
IPAH men & $18 / 25(72.0 \%)$ & $9 / 25(36.3 \%)$ & $12 / 25(48.0 \%)$ \\
CTEPH men & $48 / 48(100.0 \%)$ & $19 / 48(39.6 \%)$ & $23 / 48(47.9 \%)$ \\
& $\mathrm{p}<0.001$ & $\mathrm{p}=0.80$ & $\mathrm{p}=1.0$ \\
IPAH+BMPR2 mutations & $7 / 10(70.0 \%)^{*}$ & $4 / 10(40.0 \%)^{*}$ & $2 / 10(20.0 \%)^{*}$
\end{tabular}

*Unable to calculate a p value as no comparable group of CTEPH patients with BMPR2 mutations.

BMPR2, bone morphogenetic protein receptor type 2; CTEPH, chronic thromboembolic pulmonary hypertension; ERA, endothelin receptor antagonist; IPAH, idiopathic pulmonary arterial hypertension. 
Table 3 Point prevalence of unexplained iron deficiency in matched IPAH and CTEPH patients

\begin{tabular}{|c|c|c|c|c|c|}
\hline Point prevalence of iron deficiency & CTEPH & $\begin{array}{l}\text { IPAH (including } \\
\text { BMPR2 mutations) }\end{array}$ & $\begin{array}{l}\text { p Value } \\
\text { (vs CTEPH) }\end{array}$ & $\begin{array}{l}\text { IPAH (excluding } \\
\text { BMPR2 mutations) }\end{array}$ & $\begin{array}{l}\text { p Value } \\
\text { (vs CTEPH) }\end{array}$ \\
\hline All & $6 / 122(4.9 \%)$ & $26 / 85(30.1 \%)$ & $<0.0001$ & $20 / 75(26.7 \%)$ & $<0.0001$ \\
\hline Premenopausal women & $2 / 26(7.7 \%)$ & $13 / 26(50.0 \%)$ & 0.002 & $10 / 22(45.5 \%)$ & 0.006 \\
\hline Men & $1 / 48(2.1 \%)$ & $7 / 25(28.0 \%)$ & 0.002 & $4 / 19(21.0 \%)$ & 0.02 \\
\hline IPAH+BMPR2 mutations & N/A & $6 / 10(60.0 \%)^{*}$ & N/A & $\mathrm{N} / \mathrm{A}$ & $\mathrm{N} / \mathrm{A}$ \\
\hline
\end{tabular}

*Unable to calculate a $\mathrm{p}$ value as no comparable group of CTEPH patients with BMPR2 mutations.

BMPR2, bone morphogenetic protein receptor type 2; CTEPH, chronic thromboembolic pulmonary hypertension; IPAH, idiopathic pulmonary arterial hypertension.

Mann-Whitney test. Correlations between the iron indices, IL-6 and hepcidin levels were calculated using the Spearman method. Finally, linear regression was used to construct a representative relationship between significantly correlated factors and the relationship derived from patients with idiopathic $\mathrm{PAH}$ was compared with that of patients with CTEPH.

\section{RESULTS}

\section{Description of PAH and CTEPH groups}

The baseline characteristics and haemodynamics of the PAH and CTEPH groups as a whole and for the subgroups are shown in tables 1 and 2 . The patients with PAH were younger than those with CTEPH. There was no significant difference in the right atrial pressures or cardiac indices between any of the IPAH and CTEPH subgroups. Premenopausal IPAH women and men had significantly worse mean PA pressure and pulmonary vascular resistance than their CTEPH counterparts but comparable 6MWD.

The proportion of patients on anticoagulation was higher in patients with CTEPH than in those with PAH. The proportion of patients who had saturations of $<95 \%$ on air was comparable overall and for the premenopausal women and men subgroups. The proportion of patients on endothelin receptor antagonists was either similar or less in all PAH groups than in the corresponding CTEPH groups. Six of 13 iron-deficient premenopausal women with IPAH either reported menorrhagia or had a record of heavy menstrual loss in their case notes.

\section{Point prevalence of iron deficiency in PAH and CTEPH groups}

Twenty-six of 85 (30.1\%) patients with $\mathrm{PAH}$ were iron-deficient compared with 6 of 122 (4.9\%) patients with CTEPH

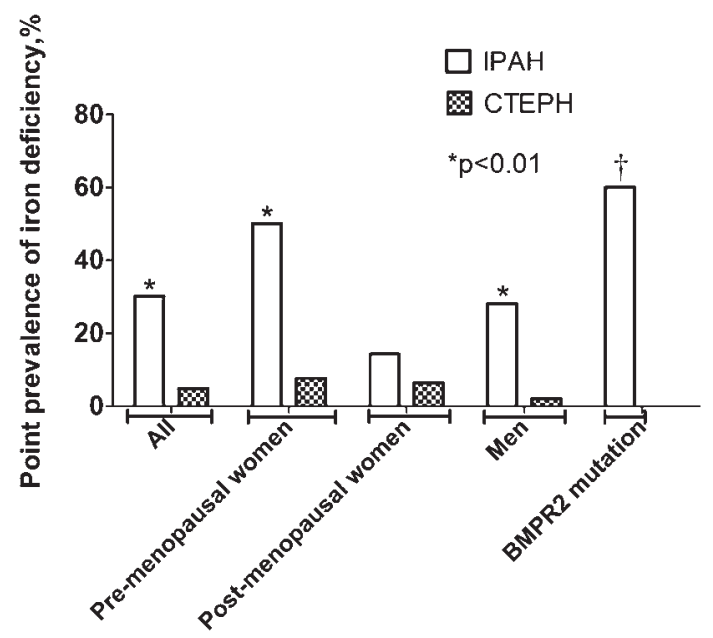

Figure 2 Point prevalence of unexplained iron deficiency in patients with IPAH and CTEPH. * $p<0.05$ vs corresponding CTEPH group. † Unable to calculate a p-value as no comparable group of CTEPH patients with BMPR2 mutations BMPR2, bone morphogenetic protein type 2 receptor; CTEPH, chronic thromboembolic pulmonary hypertension; IPAH, idiopathic pulmonary arterial hypertension. $(\mathrm{p}<0.0001)$. Thirteen of $26(50.0 \%)$ women with premenopausal idiopathic PAH were iron-deficient with no obvious cause. This was significantly different $(p=0.002)$ from their CTEPH counterparts who had a point prevalence of iron deficiency of $7.7 \%$. A similar pattern was found in the men with idiopathic PAH who had a point prevalence for iron deficiency of $28.0 \%$ compared with $2.1 \%$ in men with CTEPH $(p=0.002)$. The difference in postmenopausal women was not as marked (14.3\% in IPAH vs $6.5 \%$ in CTEPH) and this did not reach statistical significance $(p=0.16)$. The highest point prevalence of iron deficiency was found in patients with PAH carrying BMPR2 mutations ( 6 of 10 patients, $60 \%$ ). These results are summarised in table 3 and figure 2. For comparison, the prevalence of iron deficiency in the general population is reported to be $7.3-12.3 \%$ for premenopausal women, $4.7-8.4 \%$ for postmenopausal women and $2.1-3.8 \%$ for men. ${ }^{24} 25$ This analysis included patients with BMPR2 mutations. However, when patients with BMPR2 mutations were excluded, premenopausal women and men with idiopathic PAH still had a higher point prevalence of iron deficiency than those with CTEPH (table 3 ).

The multivariable model identified the type of pulmonary hypertension and the state of being a premenopausal woman as having the most impact on the presence or absence of iron deficiency. If all other explanatory variables were held fixed, changing the cause of pulmonary hypertension from CTEPH to idiopathic PAH increases the risk of being deficient in iron by $52.9 \%$. Being a premenopausal woman increases the risk of being iron-deficient by $24.0 \%$ if all other variables are held fixed at the middle of their ranges.

Twenty-three of 32 (72\%) of the iron-deficient patients were anaemic compared with 6 of 151 (4\%) non-deficient patients. Patients with iron deficiency had a mean haemoglobin of $10.4 \pm 1.0 \mathrm{~g} / \mathrm{dl}$ (normal range 13.0-18.0 g/dl for men and $11.5-16.5 \mathrm{~g} / \mathrm{dl}$ for women), an average mean corpuscular volume of 77.2 $\pm 11.0 \mathrm{fL}$ (normal range 77.0-95.0 fL) and a mean corpuscular haemoglobin concentration of $25.8 \pm 3.9 \mathrm{~g} / \mathrm{dl}$ (normal range 27.0-32.0 g/dl).

Comparison of iron-deficient and iron-replete patients with PAH The iron-deficient patients were younger than the iron-replete patients $(46 \pm 15$ vs $55 \pm 16$ years, $\mathrm{p}=0.02$ ). There were no differences between groups in right atrial pressures, cardiac indices, PVR, 6MWD or the proportion of patients who were hypoxic, antinuclear antibody-positive or treated with endothelin receptor antagonists (table 4). There was a trend towards a higher mean PA pressure in the iron-deficient patients but this did not reach significance (56 \pm 11 vs $50 \pm 14 \mathrm{~mm} \mathrm{Hg}, \mathrm{p}=0.06$ ).

\section{Iron indices and humoral factors in PAH versus CTEPH}

To investigate the basis of the unexplained iron deficiency in patients with $\mathrm{PAH}$, we measured iron indices and humoral factors known to influence iron metabolism (ie, hepcidin and IL-6) ${ }^{19-22}$ in a population of 20 patients with idiopathic PAH 
Table 4 Comparison of parameters between iron-deficient and iron-replete patients with IPAH

\begin{tabular}{|c|c|c|c|}
\hline Characteristics & Iron-deficient & Iron-replete & p Value \\
\hline Number of patients & 26 & 59 & - \\
\hline Age, years & $45.5(15.4)$ & $55.2(16.1)$ & 0.02 \\
\hline RAP, mm Hg & $9.5(5.0)$ & $9.5(5.6)$ & 0.95 \\
\hline Mean PA pressure, mm Hg & $55.9(11.2)$ & $50.3(14.0)$ & 0.06 \\
\hline $\mathrm{Cl}, \mathrm{l} / \mathrm{m}^{2}$ & $2.1(0.6)$ & $1.9(0.6)$ & 0.39 \\
\hline Pulmonary vascular resistance, dynes. $\mathrm{cm}^{-5}$ & $939(408)$ & $1000(486)$ & 0.55 \\
\hline 6MWD, m & $320(122)$ & $320(115)$ & 0.97 \\
\hline Proportion ANA-positive & $3 / 26(11.5 \%)$ & $11 / 59(18.6 \%)$ & 0.54 \\
\hline Proportion with saturation $<95 \%$ on room air & $12 / 26(46.2 \%)$ & $23 / 59(40.0 \%)$ & 0.79 \\
\hline Proportion treated with ERAs & $13 / 26(50.0 \%)$ & $17 / 59(28.8 \%)$ & 0.09 \\
\hline
\end{tabular}

ANA, antinuclear antibody; $\mathrm{Cl}$, cardiac index; ERA, endothelin receptor antagonist; IPAH, idiopathic pulmonary arterial hypertension; 6MWD, 6-minute walking distance; PA, pulmonary artery; RAP, right atrial pressure.

and 24 with CTEPH. As the patients with CTEPH were chosen to match the haemodynamic indices of those with $\mathrm{PAH}$, there were no significant differences in right atrial pressure, mean PA pressure or cardiac index between these two groups (full details in table 2 in online supplement). The idiopathic PAH group tended to be iron-deficient when compared with the CTEPH group, with a lower serum iron $(15.5 \pm 6.3$ vs $23.0 \pm 11.0 \mu \mathrm{mol} / \mathrm{l}$,

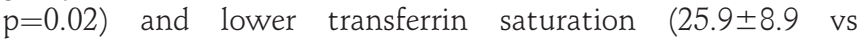
$37.4 \pm 14.8 \%, p=0.008)$. The hepcidin level in idiopathic PAH was lower but this did not quite reach statistical significance ( $25.1 \pm 18.8$ vs $39.7 \pm 27.6 \mathrm{ng} / \mathrm{ml}, \mathrm{p}=0.07)$. IL-6 levels were similar in both IPAH and CTEPH ( $4.3 \pm 3.3$ vs $4.2 \pm 2.8 \mathrm{pg} / \mathrm{ml}, \mathrm{p}=0.99$ ). Full results are shown in figure 3 .

Interestingly, the relationship between IL-6 and iron metabolism may be different in PAH and CTEPH. There was a significant correlation between iron levels and IL-6 values in idiopathic PAH $(r=-0.6, p=0.006)$ but not in CTEPH $(r=-0.36$, $\mathrm{p}=0.09$ ). Similarly, there was a correlation between IL-6 levels and transferrin saturations in PAH $(r=-0.68, p=0.001)$ but not in CTEPH ( $\mathrm{r}=-0.25, \mathrm{p}=0.27)$. When this is plotted graphically, it becomes apparent that in general, for a given value of IL-6, the corresponding iron value and transferrin saturation is lower in the PAH group than in the CTEPH group (figure 4). IL-6 is generally thought to act on iron metabolism via hepcidin, which negatively regulates iron absorption and recycling. ${ }^{20-22}$ However, higher IL-6 levels are associated with increased hepcidin in CTEPH but not in PAH (figure 4).

\section{DISCUSSION}

This is the first study to demonstrate an association between iron deficiency and idiopathic PAH. This is particularly marked

Figure 3 Iron indices and humoral factors affecting iron metabolism in matched IPAH and CTEPH patients. CTEPH, chronic thromboembolic pulmonary hypertension; IL-6, interleukin-6; IPAH, idiopathic pulmonary arterial hypertension; \%TSAT, \% transferrin saturation; UIBC unsaturated iron binding capacity.
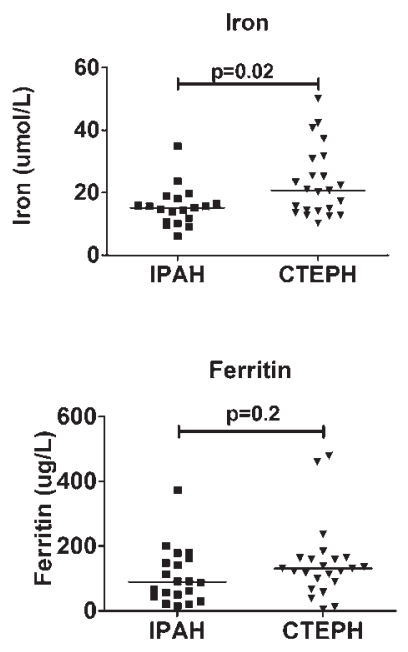

in premenopausal women and patients with heritable $\mathrm{PAH}$ harbouring BMPR2 mutations. Iron deficiency is well recognised in patients with PAH secondary to congenital heart disease. ${ }^{1} 2$ This is usually associated with polycythaemia and the underlying mechanism is thought to be hypoxia-driven secondary erythropoiesis. Anaemia is also common in left heart failure. ${ }^{56}$ There are several theories as to why this might be the case. First there is an inflammatory milieu associated with heart failure, and increased levels of cytokines such as IL- 6 could drive an 'anaemia of chronic inflammation'. ${ }^{26}$ Chronic left heart failure is also associated with a general dysfunction of both myelopoietic and erythropoietic lineages, with both reduced colony-forming potential and increased apoptosis. ${ }^{27}$ Haematinic deficiencies including $\mathrm{B}_{12}$, folate and iron deficiencies have also been detected in $6 \%, 8 \%$ and $13 \%$, respectively, of a group of anaemic patients with left heart failure. ${ }^{6}$ It is also possible that advanced heart failure leads to generalised gut oedema which prevents iron absorption.

We considered possible explanations for iron deficiency in our patients. The proportion of patients with saturations $<95 \%$ on air was comparable in the PAH and CTEPH groups (41\% vs $50 \%$, $\mathrm{p}=0.26$ ) while patients in the PAH group were iron-deficient to a greater degree. We also compared haemodynamic indices between iron-deficient and iron-replete patients since advanced right heart failure might lead to gut oedema and iron malabsorption. However, there was no difference in right atrial pressures or cardiac indices between these two groups.

An inflammatory basis for the iron deficiency and anaemia in $\mathrm{PAH}$ seems likely as it has previously been demonstrated that patients with PAH have increased levels of IL- $6{ }^{28}$ Indeed, we have shown that IL-6 levels predict mortality in these patients. ${ }^{29}$
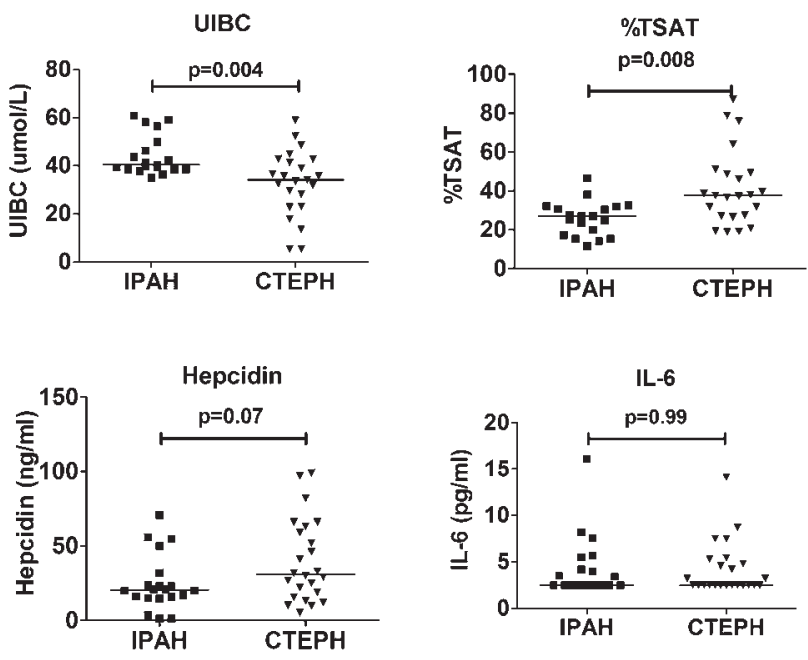
Figure 4 Relationship between IL-6 and iron indices in IPAH and CTEPH patients. CTEPH, chronic thromboembolic pulmonary idiopathic pulmonary arterial hypertension; \%TSAT, \% transferrin saturation; UIBC, unsaturated iron binding capacity. hypertension; IL-6, interleukin-6; IPAH
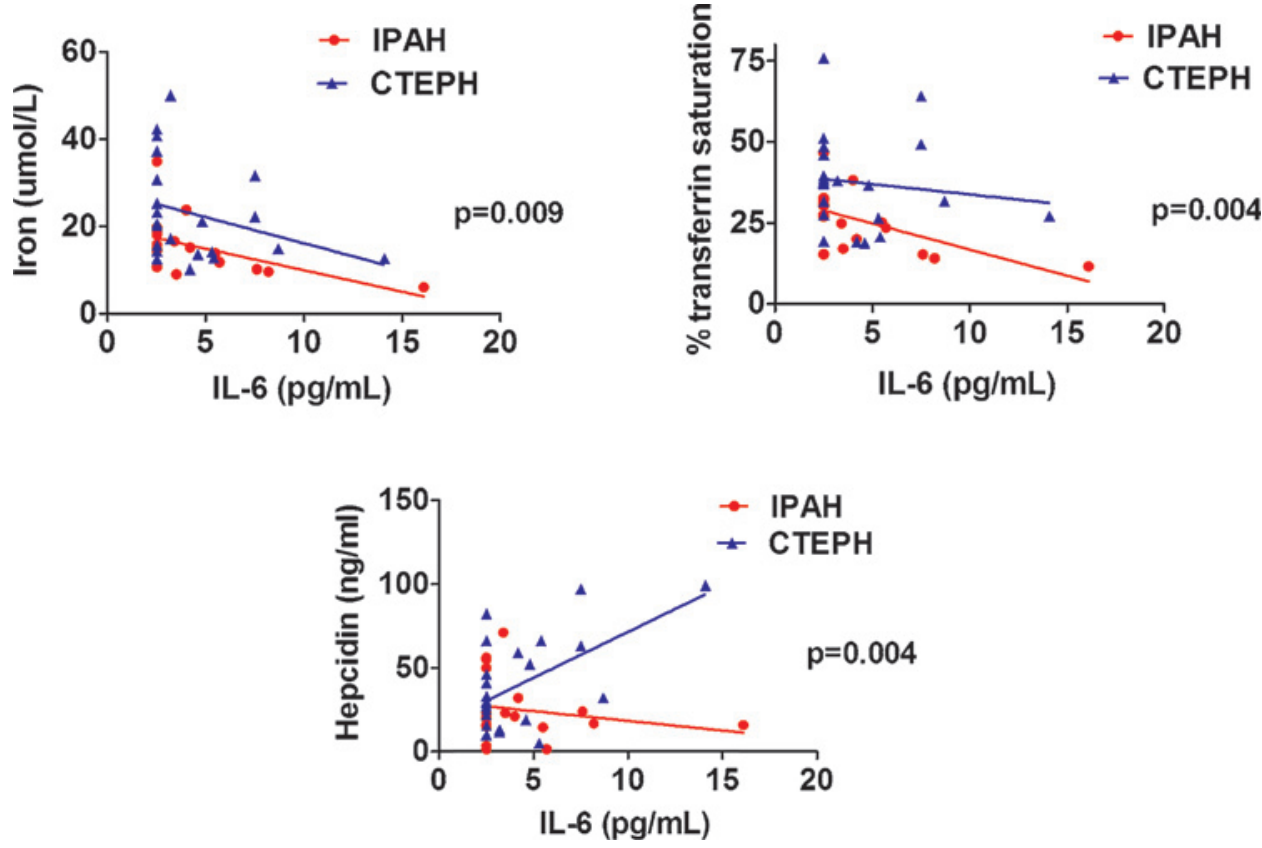

IL-6 can stimulate production of hepcidin, the principal hormone governing iron homeostasis, in vitro and in vivo. ${ }^{19} \mathrm{We}$ have shown that, for a given value of IL-6, the corresponding iron value is lower in the PAH group than in the CTEPH group. However, higher IL-6 levels are associated with increased hepcidin in CTEPH but not in PAH. This leads us to postulate that the effect of IL-6 is uncoupled from hepcidin regulation in PAH compared with CTEPH, either due to an increased sensitivity on the part of the PAH patients or that IL-6 is acting via a different mechanism. Hepcidin has recently been shown to act via a BMP-dependent pathway. ${ }^{13} 14$ The fact that patients with BMPR2 mutations have the highest point prevalence of iron deficiency might suggest an interaction between the loss of BMPR2 function, IL- 6 and iron metabolism.

The principal limitation of the prevalence study lies in its retrospective design. As a result, there was no set protocol for investigating iron deficiency and the onus was on the referring medical teams to investigate underlying causes.

Finally, iron deficiency may have a previously unrecognised impact on the symptoms and prognosis of these patients. The French respiratory home care network, the Association Nationale pour le Traitement à Domicile de l'Insufficance Respiratoire Chronique, has shown an association between low haemoglobin levels and mortality in patients with chronic obstructive pulmonary disease. ${ }^{30}$ Anaemia is also shown be associated with increased hospitalisation rates and worse mortality rates in patients with left heart failure. ${ }^{3-5} \mathrm{~A}$ Cochrane review covering erythropoiesis-stimulating agents in 794 patients with chronic heart failure has shown lower mortality and better exercise tolerance with no increase in adverse events in the treatment groups. ${ }^{10}$ Recently, Krasuski et al have demonstrated a link between anaemia and poorer survival in a group of patients with pulmonary hypertension of mixed aetiologies. ${ }^{31}$ Our study highlights the prevalence of iron deficiency in $\mathrm{PAH}$, and it would seem prudent to recommend screening for iron deficiency in these patients. It remains to be determined what the optimal method of iron replacement will be in these patients and whether such an intervention would significantly impact on symptoms or survival.

\section{CONCLUSIONS}

The prevalence of iron deficiency is significantly higher in patients with idiopathic and heritable PAH than in a comparable group of patients with CTEPH. This is not attributable to warfarin or endothelin receptor antagonist use and may be linked to IL-6. Further investigation is needed to elucidate the mechanism of iron deficiency in these patients and to determine whether iron supplementation will be beneficial.

Acknowledgements Ms Denise Hodgkins was responsible for setting up the patient database. Ms Natalie Doughty assisted in data collection.

Funding This work was partly funded by an MRC (UK) (to ES) Research Training Fellowship, the British Heart Foundation, the Cambridge NIHR Biomedical Research Centre and the Sackler studentship.

Competing interests ES has received travel grants from Encysive and GlaxoSmithKline and an unrelated unrestricted research grant from Pfizer. CMT has received travel grants from Actelion. MRT has received travel grants from Encysive and GlaxoSmithKline. RMR has received travel grants from Pfizer and GlaxoSmithKline. KKS has received honoraria from Encysive Pharmaceuticals for an advisory board meeting and travel grants from Actelion, United Therapeutics Corporation, Encysive and GlaxoSmithKline. NWM has received honoraria for educational talks from Actelion and Pfizer and a research grant from Novartis. JP-Z has received honoraria from Actelion, Pfizer, GlaxoSmithKline, Encysive and Schering for speaking at conferences and advisory board meetings. She also holds a joint grant of $£ 75 \mathrm{~K}$ between Actelion, Pfizer, Schering and United Therapeutics. VM, MB and JA have no competing interests.

Ethics approval This study was conducted with the approval of the Cambridgeshire 3 Research Ethics Committee.

Provenance and peer review Not commissioned; externally peer reviewed.

\section{REFERENCES}

1. Deanfield J, Thaulow E, Warnes C, et al; Task Force on the Management of Grown Up Congenital Heart Disease, European Society of Cardiology; ESC Committee for Practice Guidelines. Management of grown up congenital heart disease. Eur Heart $J$ 2003;24:1035-84.

2. Broberg CS, Bax BE, Okonko DO, et al. Blood viscosity and its relationship to iron deficiency, symptoms and exercise capacity in adults with cyanotic congenital heart disease. J Am Coll Cardiol 2006;48:356-65.

3. McMurray JJ. What are the clinical consequences of anemia in patients with chronic heart failure? J Card Fail 2004;10(1 Suppl):S10-12.

4. Horwich TB, Fonarow GC, Hamilton MA, et al. Anemia is associated with worse symptoms, greater impairment in functional capacity and a significant increase in mortality in patients with advanced heart failure. J Am Coll Cardiol 2002;39:1780-6. 
5. Jankowska EA, Rozentryt P, Witkowska A, et al. Iron deficiency: an ominous sign in patients with systolic heart failure. Eur Heart J 2010;15:1872-80.

6. Witte KK, Desilva R, Chattopadhyay S, et al. Are hematinic deficiencies the cause of anemia in chronic heart failure? Am Heart J 2004;147:924-30.

7. Anker SD, Comin Colet J, Filippatos G, et al; FAIR-HF Trial Investigators. Ferric carboxymaltose in patients with heart failure and iron deficiency. N Engl J Med 2009; $361: 2436-48$

8. Lane KB, Machado RD, Pauciulo MW, et al. Heterozygous germline mutations in BMPR2, encoding a TGF-beta receptor, cause familial primary pulmonary hypertension. The International PPH Consortium. Nat Genet 2000;26:81-4

9. Deng Z, Morse JH, Slager SL, et al. Familial primary pulmonary hypertension (gene $\mathrm{PPH} 1)$ is caused by mutations in the bone morphogenetic protein receptor-II gene. Am J Hum Genet 2000:67:737-44.

10. Lin L, Valore EV, Nemeth $\mathrm{E}$, et al. Iron transferring regulates hepcidin synthesis in primary hepatocyte culture through hemojuvelin and BMP2/4. Blood 2007;110:2182-9.

11. Babitt JL, Huang FW, Xia Y, et al. Modulation of bone morphogenetic protein signalling in vivo regulates systemic iron balance. J Clin Invest 2007;117:1933-9.

12. Ngo K, Kotecha D, Walters JA, et al. Erythropoiesis-stimulating agents for anaemia in chronic heart failure patients. Cochrane Database Syst Rev 2010;(1):CD007613. doi:10.1002/14651858. CD007613.pub2.

13. Ma AG, Schouten EG, Sun YY, et al. Supplementation of iron alone and combined with vitamins improves haematological status, erythrocyte membrane fluidity and oxidative stress in anaemic pregnant women. Br J Nutr 2010;104:1655-61.

14. Smith TG, Talbot NP, Privat C, et al. Effects of iron supplementation and depletion on hypoxic pulmonary hypertension: two randomized controlled trials. JAMA 2009;302:1444-50.

15. Simonneau G, Robbins IM, Beghetti M, et al. Updated clinical classification of pulmonary hypertension. J Am Coll Cardiol 2009;54(1 Suppl):S43-54.

16. Dingemanse J, Van Giersbergen PLM. Clinical pharmacology of bosentan, a dual endothelin receptor antagonist. Clin Pharmacokinet 2004;43:1089-115.

17. Barst RJ, Langleben D, Badesch D, et al; STRIDE-2 Study Group. Treatment of pulmonary arterial hypertension with the selective endothelin-A receptor antagonist sitaxsentan. J Am Coll Cardiol 2006:47:2049-56.

18. Baynes RD. Assessment of iron status. Clin Biochem 1996;29:209-15.
19. Wrighting DM, Andrews NC. Interleukin-6 induces hepcidin expression through STAT3. Blood 2006;108:3204-9.

20. Nicolas G, Bennoun M, Devaux I, et al. Lack of hepcidin gene expression and severe tissue iron overload in upstream stimulatory factor 2 (USF2) knockout mice. Proc Natl Acad Sci U S A 2001;98:8160-2.

21. Pigeon C, llyin G, Courselaud B, et al. A new mouse liver-specific gene, encoding a protein homologous to human antimicrobial peptide hepcidin, is overexpressed during iron overload. J Biol Chem 2001;276:7811-19.

22. Nicolas G, Bennoun $M$, Porteu $A$, et al. Severe iron deficiency anemia in transgenic mice expressing liver hepcidin. Proc Natl Acad Sci U S A 2002;99:4596-601.

23. Busbridge $\mathbf{M}$, Griffiths $\mathrm{C}$, Ashby $\mathrm{D}$, et al. Development of a novel immunoassay for the iron regulatory peptide hepcidin. Br J Biomed Sci 2009:66:150-7.

24. Hirani V, Brookes M, McMunn A, et al. Anthropometry. In: Shaw A, McMunn A, Field J, eds. The Scottish Health Survey, 1998:13.8.4. http://www.sehd.scot.nhs.uk/ scottishhealthsurvey/sh8-00.html (accessed 25 Jul 2010).

25. Looker AC, Dallman PR, Carroll MD, et al. Prevalence of iron deficiency in the United States. JAMA 1997:277:973-6.

26. Kourea K, Parissis JT, Farmakis D, et al. Effects of darbepoetin-alpha on plasma pro-inflammatory cytokines, anti-inflammatory cytokine interleukin-10 and soluble Fas/Fas ligand system in anemic patients with chronic heart failure. Atherosclerosis 2008;199:215-21.

27. Westenbrink BD, Voors AA, De Boer RA, et al. Bone marrow dysfunction in chronic heart failure patients. Eur J Heart Fail 2010;12:676-84.

28. Humbert M, Monti G, Brenot F, et al. Increased interleukin-1 and interleukin-6 serum concentrations in severe primary pulmonary hypertension. Am J Respir Crit Care Med 1995; 151:1628-31.

29. Soon E, Holmes AM, Treacy CM, et al. Elevated levels of inflammatory cytokines predict survival in idiopathic and familial pulmonary arterial hypertension. Circulation 2010;122:920-7

30. Chambellan A, Chailleux E, Similowski T; ANTADIR Observatory Group. Prognostic value of the hematocrit in patients with severe COPD receiving long-term oxygen therapy. Chest 2005;128:1201-8.

31. Krasuski RA, Hart SA, Smith B, et al. Association of anemia and long-term survival in patients with pulmonary hypertension. Int J Cardiol Published Online First: 14 May 2010. doi:10.1016/j.jijcard.2010.04.038.

\section{Lung alert}

\section{The inhibition of anaplastic lymphoma kinase in non-small cell lung tumours with the ALK rearrangement may result in tumour shrinkage}

In this study, the therapeutic efficacy of inhibiting anaplastic lymphoma kinase (ALK) in oncogenic fusion genes consisting of EML4 and ALK was explored in an early-phase clinical trail of crizotinib. Tumour samples from 1500 patients with non-small cell lung cancer (NSCLC) were screened for the presence of ALK rearrangements and 82 patients with advanced ALK positive disease were identified. They were started on crizotinib $250 \mathrm{mg}$ twice daily in 28-day cycles.

Results showed that patients with ALK rearrangements were younger than those without the rearrangements and most patients had little or no exposure to tobacco.

At the mean treatment duration of 6.4 months, the overall response rate was $57 \%$ and $33 \%$ had stable disease. A total of 63 of the 82 patients continued to receive crizotinib at the time of data cut-off, and the estimated probability of a 6-month progression-free survival was $72 \%$.

The inhibition of ALK in lung tumours with the ALK rearrangement resulted in tumour shrinkage or stable disease in most patients, providing a case for genotyping in lung cancer. However, this arrangement is only seen in 5\% of NSCLC.

Kwak EL, Bang Y, Camidge R, et al. Anaplastic lymphoma kinase inhibition in non-small-cell lung cancer. N Engl J Med 2010;363:1693-703.

\section{Louise Hancock}

Correspondence to Dr Louise Hancock, North Middlesex Hospital, London, UK; Ihancock@doctors.org.uk

Published Online First 10 February 2011

Thorax 2011;66:332. doi:10.1136/thx.2011.159467 